\title{
UTILIZACIÓN DE LOS ORDENADORES EN LA ENSEÑANZA DE LAS CIENCIAS
}

\author{
VALDÉS CASTRO, R. y VALDÉS CASTRO, P. \\ Facultad de Física. Instítuto Superior Pedagógico Enrique José Varona. \\ Ciudad Libertad. Marianao. Ciudad de la Habana. Cuba.
}

\section{SUMMARY}

The object of this article is to discuss the relevance of the use of microcomputers in science teaching. The authors consider that using microcomputers in order to let students get familiar with the methodological changes they have caused in contemporary scientific-technical activity, could produce radical transformations in science teaching.

\section{INTRODUCCIÓN}

El empleo de los ordenadores en la enseñanza de las ciencias es una realidad (Abreu, Fábrega y Oliveró 1991, Barberá y Sanjosé 1990, Herrán, Parrilla et al. 1992, Keeport 1990, Valdés, Valdés, Clavelo y Carrillo 1992, etc.). En la actualidad las discusiones se centran alrededor de la cuestión đe cuáles son el alcance y las perspectivas que tiene la introducción de esta nueva tecnología en la educación (Gil 1991).

¿Constituye la utilización de ordenadores en la enseñanza de las ciencias una innovación sustancial o es sencillamente un medio didáctico como pueden ser el cine y la televisión?

La respuesta a la pregunta anterior depende de los fines con que se utilicen los ordenadores. Si se emplean simplemente como medio đidáctico (medio audiovisual, tutorial, sistema evaluativo), entonces, aunque ello permite elevar la productividad de la enseñanza, no conduce a una transformación radical, pues no afecta ni sus objetivos, ni su contenido, sino apenas sus métodos. Sin embargo, si los ordenadores se utilizan con el fin de familiarizar a los estudiantes con los cambios metodológicos que se han producido en la actividad científicotécrica contemporánea, entonces su utilización puede llegar a representar una revolución, en la medida que conduzca a transformaciones sustanciales de los objetivos, el contenido y los métodos de enseñanza.

\section{IMPACTO METODOLÓGICO DE LOS ORDENADORES EN LA CIENCIA}

La función histórica esencial de los medios técnicos ha sido la sustitución del hombre en sus labores, con la consecuente elevación de la efectividad del trabajo. Hasta mediados del siglo xx la creación de esos medios estaba fundamentalmente encaminada a liberar al hombre del trabajo meramente físico. Por el contrario, en la actualidad lo característico es la invención de máquinas capaces de realizar acciones intelectuales: búsqueda, almacenamiento y transmisión de información, cálculos, mediciones, reconocimiento de imágenes, control de procesos productivos, pronósticos. Uno de los principales eslabones de esta revolución tecnológica es el ordenador, que ha penetrado en los más diversos campos de la vida; desde la ciencia hasta las labores domésticas.

Los ordenadores electrónicos digitales son en gran medida un resultado de los logros de la ciencia de nuestro siglo; pero al propio tiempo han variado sustancialmente los métodos de investigación e influido decisivamente en el desarrollo científico.

Hoy los ordenadores tienen múltiples funciones, pero el objetivo inicial con que fueron creados a mitad de siglo fue servir de medio de cálculo. La ciencia del siglo pasado construía los modelos matemáticos de los fenómenos fundamentalmente mediante ecuaciones que tienen solución analítica. En cambio, gracias a la invención 
de los ordenadores, en la actualidad es habitual describir los fenómenos mediante ecuaciones cuyas soluciones se hallan con el empleo de métodos numéricos. El progreso de ramas de vanguardia de la ciencia como la cosmonáutica, la física de las partículas elementales y del estado sólido, la química cuántica y la microelectrónica serían imposibles sin el empleo de los métodos numéricos.

El desarrollo de la microelectrónica en la década del 60 condujo al incremento de la velocidad en el procesamiento de la información por los ordenadores digitales y en especial, a la posibilidad de que esas máquinas controlaran al mismo tiempo teclados, displays, impresoras y otros equipos. Estos cambios tecnológicos posibilitaron la automatización de experimentos mediante ordenadores y el régimen de diálogo en la comunicación hombre-máquina.

La flexibilidad en la interacción hombre-máquina permití la amplia realización de experimentos con modelos matemáticos en el ordenador o simulación matemática.

La simulación matemática guarda semejanza con la investigación experimental y con la investigación teórica. Como en el experimento real, durante la simulación se examina un sistema variando sus propiedades y situándolo en diferentes condiciones.

Esto se logra, por ejemplo, cambiando los valores de las constantes y de las condiciones iniciales de las ecuaciones diferenciales con que se describe un proceso. Pero independientemente de las acciones que realice el investigador, él no estudia directamente un sistema material, sino ideal y el análisis efectuado es eminentemente teórico. La experimentación con modelos matemáticos en el ordenador es uno de los aportes de la ciencia contemporánea a la investigación científica.

La creación de las técnicas de inteligencia artificial a partir de la década del 70 condujo a la elaboración de softwares capaces de hallar la solución analítica de problemas matemáticos y a la creación de sistemas expertos. Estos medios permitieron liberar a los investigadores de la realización de complejas transformaciones algebraicas y orientarlos en la búsqueda de la solución a los problemas planteados.

Gracias a la invención de los microordenadores, las transformaciones operadas en los métodos del trabajo científico han adquirido un carácter masivo; actualmente pueden encontrarse potentes ordenadores no sólo en salas especializadas de las instituciones científicas, sino también en la mesa de trabajo de cualquier investigador, profesor e incluso de muchos estudiantes.

\section{NUEV OS OBJETIVOS Y CONTENIDO DE LA ENSENANZA DE LAS CIENCIAS}

Es tal el efecto de los ordenadores en la sociedad, que hoy se habla de la necesidad de crear una cultura informática en grandes sectores de la población. Ya desde la enseñanza primaria, los niños utilizan juegos didácticos que los familiarizan con el ordenador y luego adquieren los conocimientos básicos para la solución de algunos problemas empleando ese medio técrico.

En general, como resultado de la utilización de los ordenadores, la escuela moderna ha logrado graduar alumnos con conocimientos sobre la construcción y el funcionamiento de los ordenadores digitales, con habilidades para elaborar algoritmos y con hábitos para el uso de bases de datos y de paquetes de programas como los procesadores de textos, programas de gráficos y hojas de cálculo. Ha cambiado sustancialmente el ambiente de la enseñanza escolar.

Muchos estudiantes concluyen hoy la enseñanza preuniversitaria con los hábitos necesarios para confeccionar sus propios programas; aunque, a decir verdad, la confección de esos programas no suele familiarizarlos con el cambio metodológico que representó el empleo de los ordenadores en la ciencia.

Tomando en consideración el impacto que han tenido los ordenadores en la ciencia y lo que en esta dirección ya se ha logrado en la esfera de la educación, es posible plantear entre los objetivos fundamentales de la enseñanza de las ciencias los siguientes:

- Formar conocimientos, habilidades y hábitos necesarios para utilizar el ordenador como medio de cálculo en la resolución de problemas.

- Formar conocimientos, habilidades y hábitos necesarios para la realización de experimentos con modelos matemáticos en el ordenador.

- Relacionar a los alumnos con los prinicipios tecnológicos de la automatización de los experimentos.

- Familiarizar a los estudiantes con el empleo de programas inteligentes.

Los problemas que se resuelven utilizando el ordenador como medio de cálculo pueden ser de dos tipos: aquéllos cuya solución se halla simplemente mediante la sustitución de valores en alguna fórmula $y$ aquéllos que requieren la aplicación de los métodos numéricos. En el nivel preuniversitario es posible relacionar a los alumnos con los métodos numéricos de diferenciación, integración, cálculo de extremos de funciones, resolución de ecuaciones diferenciales y ajuste de curvas. Con este fin ya se ha acumulado abundante material docente en la literatura pedagógica especializada (Frank y Kluk 1990, Roller y Blum 1981, Roller y BIum 1982).

La realización de experimentos de simulación en el ordenador debe contribuir a esclarecer el concepto de modelo matemático y la esencia del método empleado. Actualmente, debido a la utilización de los ordenadores en la ciencia, el concepto de modelo matemático se ha ampliado y comprende, además de la representación de fenómenos mediante funciones y ecuaciones, la representación de procesos con algoritmos de cúlculo. De ahí la necesidad de incluir en la actividad de los alumnos no solamente la experimentación con ecuaciones, como las 
del Ianzamiento de proyectiles (Keeport 1990), sino también el trabajo con algoritmos de simulación, como los de modelación matemática de fenómenos aleatorios (Olson 1990).

El hecho de que los alumnos conozcan los principios tecnológicos de la automatización de experimentos (Herrán, Parrilla et al. 1992, Vaidés, Valdés, Clavelo y Carrillo 1992) les hace asequible el conocimiento de las bases del control automatizado de los procesos productivos y les permite mostrar la estrecha relación que hay entre ciencia, técnica y sociedad. Ello presupone el conocimiento de una serie de dispositivos como sensores, puertos de entrada-salida, convertidor analógicodigital, interfaz de conexión, etc. Incluye la comprensión de la representación binaria del estado de los dispositivos electrónicos y el estudio de los operadores lógicos NOT, AND, OR y XOR. También supone que los alumnos trabajen con instalaciones experimentales tan sencillas como sea posible, que permitan poner claramente de manifiesto cómo ocurre la selección, transmisión, almacenamiento y procesamiento de la información durante las mediciones. En este sentido las instalaciones «caseras» tienen gran utilidad, pues son relativamente simples y posibilitan que los propios estudiantes puedan fabricarlas y logren entonces comprender, mejor y más activamente, las bases del experimento automatizado.

La familiarización de los estudiantes con el empleo de programas inteligentes (Waterman 1986) requiere aclarar: qué diferencia este tipo de software de otros, qué es un sistema experto y resolver algunos problemas que pongan de manifiesto la capacidad de esos medios para realizar transformaciones algebraicas, hallar la solución analítica de ecuaciones e integrales y, en régimen de điálogo, brindar con determinado grado de verosimilitud la solución a un problema. Por ejemplo, con sistemas expertos puede determinarse la composición química de un material desconocido a partir del análisis espectral, hallar momentos de inercia y resolver otros problemas de mecánica.

Varios años de experiencia docente en institutos preuniversitarios especializados en la enseñanza de las ciencias permiten afirmar que, en ese nivel, los alumnos son capaces de resolver exitosamente problemas propuestos con el fin de lograr los objetivos planteados.

En general, las etapas de resolución de esos problemas son:

- Creación de una situación problemática y análisis cualitativo de ella.

- Búsqueda de la estrategia de solución.

- Resolución propiamente dicha del problema.

- Análisis de los resultados obtenidos.

Como norma, la situación problemática era expresada de forma abierta; por ejemplo: ¿ cómo medir la velocidad de un móvil empleando un ordenador? Pero, en cambio, no se preguntaba: ¿cómo medir la velocidad de un carrito utilizando los puertos de juego del ordenador, dos fototransistores situados a una distancia fija uno del otro y dos linternas? El planteamiento abierto del problema era acompañado de una reflexión acerca de la importancia de su resolución.

El análisis cualitativo de la situación consistía en la representación mental y global del proceso analizado, haciendo las consideraciones necesarias para modelarlo y reflejarlo gráficamente en un esquema. Así, al plantear la necesidad de determinar el coeficiente de rendimiento de un disparo de fusil, se hacía el esquema del cañón del fusil, del proyectil en su interior y de la cámara con los vapores debidos a la combustión de la pólvora. Se consideraba que el cañón y el proyectil eran cilíndricos y que éstos hacían las veces de émbolo y pistón respectivamente.

La búsqueda de la estrategia de solución de los problemas se caracterizaba por decidir sobre las definiciones, aproximaciones, fórmulas y disposición experimental que se utilizarían. En esta etapa se determinaban las magnitudes cuyos valores numéricos exan necesarios para los cálculos y se hacía la selección de los instrumentos indispensables para efectuar las mediciones. Además, se realizaban consultas bibliográficas de orientación.

Durante la resolución propiamente dicha, los estudiantes hallaban la solución analítica de las ecuaciones planteadas, establecían el método y las fórmulas de cálculo con que habría de funcionar el ordenador, elaboraban algoritmos de programación que ellos mismos codificaban en algún lenguaje de computación conocido y, si lo exigía el problema, construían la instalación experimental necesaria.

Por último, los alumnos analizaban los resultados obtenidos, contrastándolos con la realidad y con el resultado esperado.

Rectificaban la idea inicial del programa hasta obtener un producto suficientemente terminado y planteaban nuevas situaciones a investigar.

En cada una de las etapas descritas, el profesor actuaba como organizador de la actividad de los estudiantes, a quienes orientaba mediante el diálogo y estimulaba a trabajar en equipos.

El trabajo en equipos posibilita que los alumnos expresen verbalmente sus ideas, controlen las acciones intelectuales de sus compañeros y que los miembros del colectivo, en un clima de discusión científica, se aclararen ideas aún confusas. Con tal organización de la actividad de los alumnos habra grandes posibilidades para el despliegue de la creatividad y ello se puso de manifiesto durante la confección de los programas: los equipos emulaban e intercambiaban ideas, pero ninguno elaboraba un programa igual de computación.

No es difícil comprender que la resolución de estos problemas es una tarea compleja, pues requiere de esfuerzo para la integración de conocimientos sobre infor- 
mática, matemática y la ciencia específica de que se trate, y la dedicación de numerosas horas de trabajo a la programación. De ahí que muchas veces el profesor contribuyera también a la elaboración del programa de cómputo.

Sin embargo, debe señalarse que programar independientemente no es un ejercicio intelectual de segundo orden; demanda detallar al máximo, con pensamiento profundo, las operaciones que debe ejecutar el ordenador según el lenguaje de programación empleado. Por otra parte, programar presupone actuar según una estrategia de trabajo, anticipar y obtener un efecto; comparar éste con un modelo ideal y rectificar la estrategia inicial en un proceso cíclico hasta lograr el resultado satisfactorio. Es una especie de experimento en el que predominan las acciones intelectuales. El logro de los objetivos planteados a la enseñanza de las ciencias rebasa los límites de una asignatura y requiere ajustar adecuadamente las relaciones entre las diversas disciplinas docentes. Esto último, junto al empleo de los ordenadores en su función de medio didáctico, conduce a la elevación

\section{REFERENCIAS BIBLIOGRÁFICAS}

ABREU, J., FÁBREGA, A. y OLIVERO, M., 199I. DILuz. (Ministerio de Educacion y Ciencia: Madrid).

BARBERÁ, Ó. y SANIOSÉ, V., 1990. Juegos de simulación por ordenador: un útil para la enseñanza a todos los niveles, Enseñanza de las Ciencias, 8(1), pp. 46-51.

CLACK, J. y TOEPKER, P., I990. Magnetic induction experiment, The Physics Teacher, 28(4), pp. 236-238.

FRANK, M. y KLUK, E., 1990. Equations of motion on a computer spread sheet: the damped harmonic oscillation and more, The Physics Teacher, 28(5), pp. 308-311.

GIL, D., 1991. Tendencias y experiencias innovadoras en la Enseñanza de las Ciencias. HI Taller Subrregional. (Ibercima: Santo Domingo).

HERRÁN, C., PARRILLA, J., et al., 1992. El ordenador en el laboratorio. (Ministerio de Educación y Ciencia: Madrid). de la productividad de la actividad pedagógica y posibilita evitar sobrecargas docentes al introducir el nuevo contenido de la enseñanza.

\section{CONCLUSIÓN}

E1 análisis del impacto metodológico que ha tenido la utilización de los ordenadores en el desarrollo de la ciencia y de la técnica permite formular la hipótesis de la posibilidad real de revolucionar la enseñanza. Las experiencias llevadas a cabo con grupos de alumnos del nivel preuniversitario confirman, aunque aún limitadamente, esta apreciación. La recalificación de los maestros, la elaboración de todo el material docente necesario y la elevación de la productiviđad del aprendizaje sobre la base del perfeccionamiento de los métodos y medios de enseñanza pueden garantizar el cambio radical pronosticado.
KEEPORT, D., 1990. Numerical calculation of model rocket trajectories, The Physics Teacher, 28(5), pp. 274-280.

OLSON, D., 1990. Monte Carlo Computer Simulation of a Rainbow, The Physics Teacher, 28(4), pp. 226-227.

ROLLER, D. y BLUM, A., 1981. Physics. V.1. Mechanics, waves and thermodynamics. (Holden-Day: San Francisco).

ROLLER, D. y BLUM, A., 1982. Physics. V.2. Electricity, magnetism and light. (Holden-Day: San Francisco).

VALDÉS, P., VALDÉS, R., CLAVELO, A. y CARRILLO, F., 1992. Experimentos de física utilizando un ordenador MSX y componentes del laboratorio de preuniversitario. VII Forum Nacional de Piezas de Repuesto, Equipos y Tecnologías de Avanzada.

WATERMAN, D., 1986. A guide to expert systems. (AddisonWesley Publishing Company: Massachusetts). 